\title{
ITERATIVE BLIND DECONVOLUTION METHOD \\ WITH USE OF LUCY'S ALGORITHM: \\ APPLICATION TO IR SPECKLE DATA
}

\author{
FUMIAKI TSUMURAYA \\ The Graduate University for Advanced Studies, \\ Faculty of Physics and Mathematics,Department of Astronomy, \\ Mitaka,Tokyo 181, Japan \\ and \\ NORIAKI MIURA and NAOSHI BABA \\ Hokkaido University, Faculty of Engineering, \\ Department of Applied Physics, Sapporo 060,Japan
}

\begin{abstract}
Lucy's algorithm is applied to iterative blind deconvolution method. This new approach enables to reconstruct a greatly extended object from one speckle frame and to reinforce image domain constraints. A greatly extended object makes speckle images flow over detecting surface. Lucy's algorithm is accommodated to handle such an image and built into iterative blind deconvolution methods. Computer simulation and observational data analyses are conducted and the effectiveness of the proposed methods are exemplified.
\end{abstract}

Key words: speckle - deconvolution - image reconstruction

\section{Introduction}

Iterative blind deconvolution method is a kind of successive approxmation means to solve a convolution integral equation. The algorithm is fundamentally constituted by finding symmetrical approximate solutions for each of the object and the PSF distributions. Ayers and Dainty (1988) used a modified inverse filter in spatial-frequency domain to solve the integral equation by imposing image-plane constraints for both of the object and the PSF distributions. The approximate solution modified by the constraints is adopted as an estimated distribution and the iterative process is continued. The image-plane constraints are positivity and total energy conservation. Davey et al. (1989) employed a Wiener-type filter to solve the equation. Iterative blind deconvolution method can be constructed by selecting an adequate deconvolution algorithm. The algorithms are classified into two types. The one is to conduct the deconvolution process in the spatial-frequency domain. The other is to do in the image domain.

Wiener filter conducts the process in the spatial-frequency domain as

$$
O(u)=\frac{I(u) P^{*}(u)}{|P(u)|^{2}+|N(u)|^{2} /|O(u)|^{2}},
$$

where $O(u)$ is the spectrum of an object distribution, $I(u)$ and $P(u)$ is the spectrum of the observed image and the PSF, respectively. $|N(u)|^{2}$, the noise power spectrum, should be estimated by some means.

The Lucy's algorithm conducts the process in the image domain as

$$
o^{r+1}(\xi)=o^{r}(\xi) \int_{-\infty}^{\infty} \frac{i(x)}{i^{r}(x)} p(x-\xi) d x,
$$


where $i^{r}(x)=o^{r}(x) * p(x)$. Eq.(2) satisfies the image domain constraints automatically. Tsumuraya et al. (1992) applied the algorithm to iterative blind deconvolution, and showed an image reconstruction of solar photoshere.

\section{Inflow and outflow effects}

When we observe a greatly extended object, the images usually flow over the detecting surface. In such condition, inflow and outflow of energy exist at the edge of the observed frame (fig.1).

Using Lucy's algorithm, the inflow and outflow effects can be corrected by the normalization factor for the PSF which loses a part of total probability:

$$
\alpha_{p}(\xi)=\frac{F_{p}}{\Pi(\xi / L) \star p(\xi)}
$$

where $F_{p}=\int_{\text {frame(fr.) }} p(\xi) d \xi$, and $\Pi(\xi / L)=1 \quad(-L / 2 \leq \xi \leq L / 2), 0$ (otherwise). Then the Lucy's algorithm is modified approximately by changing the infinite support of integral to the finite one, as follows

$$
o^{r+1}(\xi)=o^{r}(\xi) \int_{\text {fr. }} \frac{i(x)}{i^{r}(x)} p_{\bmod }(x \mid \xi) d x,
$$

where $p_{\bmod }(x \mid \xi)=\alpha_{p}(\xi) p(x-\xi)$, and $i^{r}(x)=\int_{\text {fr. }} o^{r}(\xi) \alpha_{p}(-\xi) p(x-\xi) d \xi$.

\section{Algorithms and their performance}

Fig. 2 shows the basic blind deconvolution scheme employing Lucy's algorithm. In this figure, S1 and S2 are the maximum values of inner iteration numbers of Lucy's algorithm, $\mathrm{k}$ and $\mathrm{K}$ are outer iteration number of blind deconvolution process and its maximum value, respectively. This scheme tends to solve the solution pair into a resemblant distribution to observed image and $\delta$-function. To avoid this difficulty, we proposed multiple blind deconvolution algorithm (fig.3:algorithm A), in which a re-initialization process for the PSF is included (Tsumuraya et al.).

On the other hand, Lucy's algorithm can be incorporated into blind deconvolution method based on the process in spatial-frequency domain (fig.4:algorithm B). This scheme completely avoids the above difficulty because of using spatialfrequency filtering, and has advantage to make image-plane constraints severe compared to the original method.

Fig. 5 shows reconstructions using these two algorithms from a simulated speckle image of the extended object affected by outflow effect. The reconstructed image using alg.A (fig.5c) is over deconvolved. Binary star reconstruction using alg.B from actual SAA data is shown in fig.7. Alg.B shows good performance. Especialy, diffraction rings can be seen in the reconstructed image.

\section{References}

Ayers, G.R. and Dainty, J.C.: 1988, Opt. Lett. 13, 547

Davey, B.L.K., Lane, R.G., Bates, R.H.T.: 1989, Opt. Commun. 69, 353

Lucy, L.B.: 1974, Astron. J. 79, 745

Tsumuraya, F., Miura, N., Baba, N.: 1992, submitted to Astron. Astrophys. , 


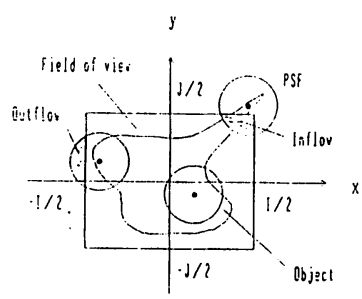

Fig. 1. Inflow and outflow effects on a speckle image

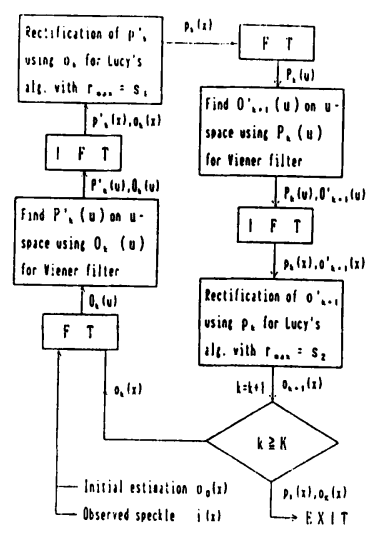

Fig. 4. New blind deconvolution scheme

Fig. 5. a) Simulated speckle image,

b) original object distribution,

c) reconstructed object by alg. A,

d) reconstructed object by alg. B.

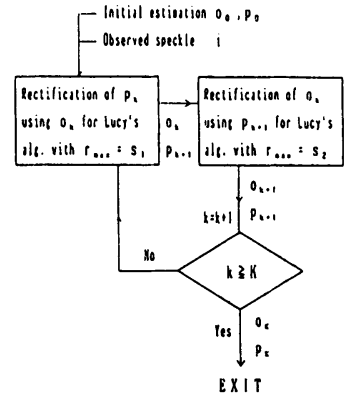

Fig. 2. Blind deconvolution scheme based on Lucy's algorithm
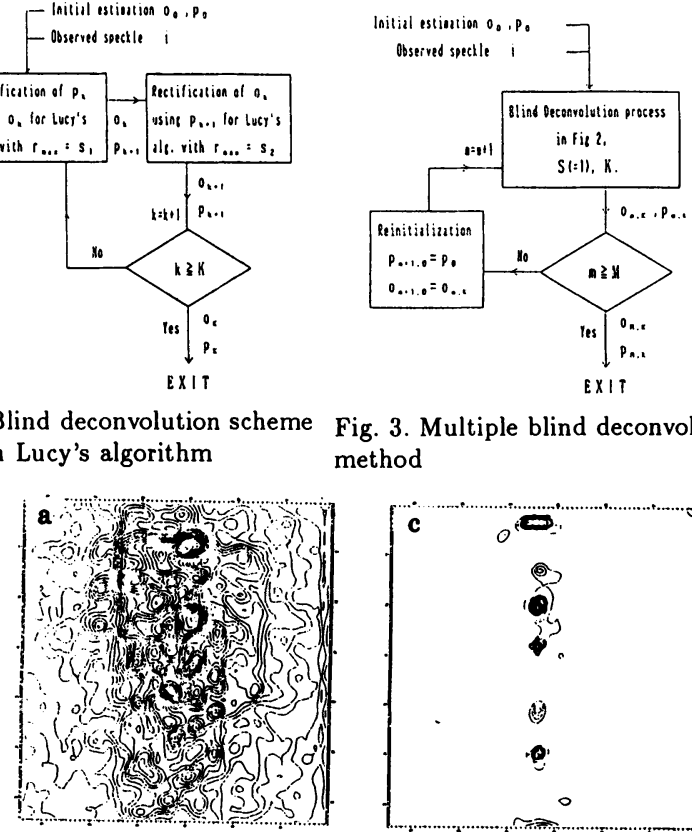

Fig. 3. Multiple blind deconvolution method
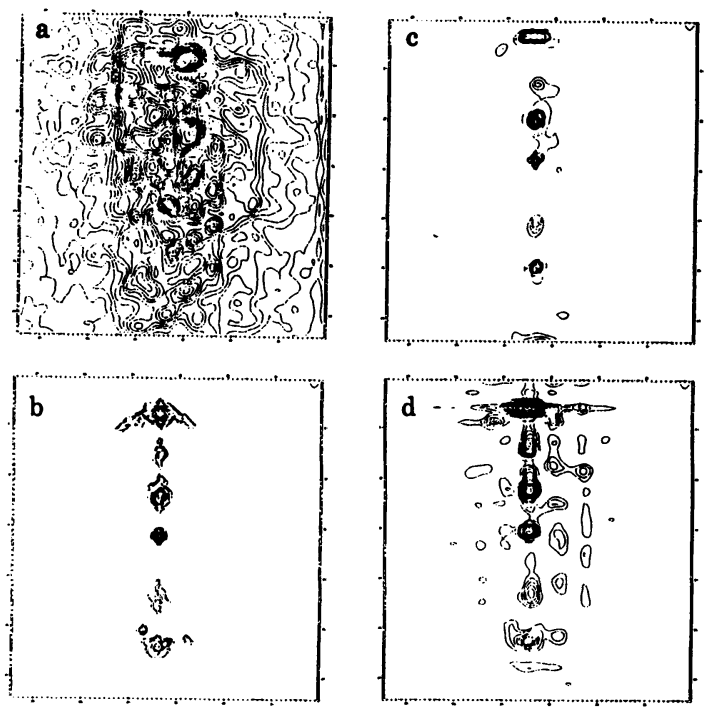
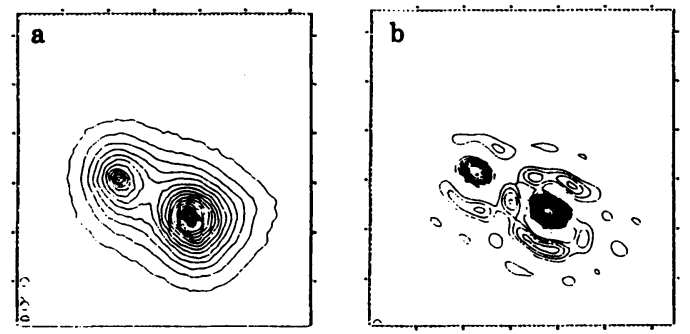

Fig. 6. a) SAA image of $\alpha \mathrm{Gem}$,

b) reconstructed object by alg. $B$.

The IR observational data $(\lambda=2.2 \mu m$, $0.17 "$ /pixel) was obtained with the $1.5 \mathrm{~m}$ telescope of CRL Koganei, Tokyo. 


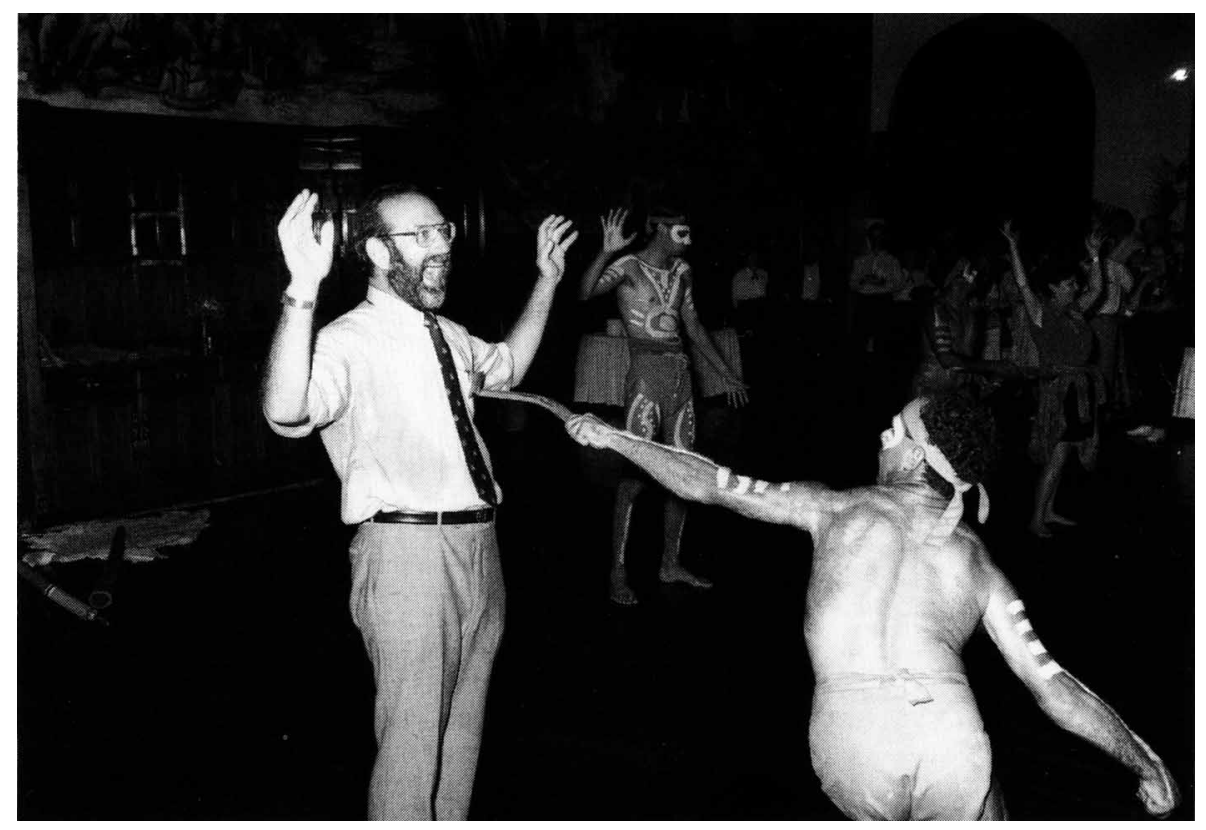

